$12^{\text {th }}$ International Congress on Mathematical Education

Regular Lectures 6-1

8 July - 15 July, 2012, COEX, Seoul, Korea

\title{
RESEARCH ON MATHEMATICS CLASSROOM PRACTICE: AN INTERNATIONAL PERSPECTIVE
}

\author{
Ah Chee Ida MOK \\ The University of Hong Kong, Hong Kong SAR, China \\ Email: iacmok@hku.hk
}

\begin{abstract}
Research on Mathematics Classroom Practice encompasses very comprehensive themes and issues that may include any studies and scientific experiments happening inside the classroom, including consideration of the key agents in the classroom (the teachers and the students), undertaken with diversified research objectives and theoretical backgrounds. To a certain extent, seeking an international perspective provides some delineation of the topic. Studies will then focus on those issues already prioritised as of interest by existing international comparative studies and those issues seen as significant within an educational system. This lecture will draw upon the work of an international project, the Learner's Perspective Study (LPS), an international collaboration of 16 countries with the aim of examining in an integrated and comprehensive fashion the patterns of participation in competently taught eighth grade mathematics classrooms.
\end{abstract}

Key words: mathematics classroom practice, cross-cultural practice, teaching strategies, learning tasks, student perspective

\section{INTRODUCTION}

Research on mathematics classroom practice encompasses very comprehensive themes and issues. Reviewing the abstracts of papers and research reports in key journals with the key word "classroom practice", a comprehensive range of presentations and discussions emerges and the broad topic of "classroom practice" can be seen to include any studies and scientific experiments happening inside the classroom, including the investigation of the key agents in the classroom (the teachers and the students), and addressing diversified research objectives and drawing on a wide range of theoretical backgrounds. To a certain extent, seeking an international perspective provides some delineation of the topic. International research offers insight into possible explanatory frameworks within which differences and similarities between cultures can be seen as offering complementary features to supplement the understanding of the nature of mathematics teaching and learning practices in one's own culture. Studies will then focus on those established as being of legitimate interest, as informed by existing international comparative studies and by the perspectives of the educational system within which each researcher is working. This lecture will draw upon the work of an international project, the Learner's Perspective Study (LPS), an international collaboration of 16 countries with the aim of examining the patterns of participation in competently taught eighth grade mathematics in an integrated and comprehensive fashion (Clarke, Kietel and Shimizu, 2006). 
The promising design and emerging findings of the project have been well received in international conferences and by the research community in education, and the LPS research community has continued to attract additional international membership. Despite their progress being at different stages, the LPS researchers from different countries greatly complement each other in terms of findings and the development of new research agenda. Some of these are reported in the publications of the LPS book series. The large body of complex data supports both the characterisation of practice in the classrooms of competent teachers and the development of theory. Participation in the LPS community provides valuable insights for understanding research on mathematics classroom practices as well as providing an opportunity for the sharing of international perspectives through the various project-specific international collaborations that form within the LPS community.

In this paper, I attempt to demonstrate the diversity of international perspectives available within the LPS research community by sharing some results taken from the first three books in the LPS series. However, I must admit that the picture will be limited to a certain extent by my personal experience and by the nature of my engagement in the project. The presentation will be divided into the following sections:

- The Learner's Perspective Study,

- Stories from the insiders: Cases with different foci,

- Making connections by comparing lesson events,

- Learning Tasks: A major vehicle in mathematics lessons, and

- Conclusion

\section{THE LEARNER'S PERSPECTIVE STUDY (LPS)}

Being constantly in a familiar environment, one usually takes things for granted and may fail to see the characteristics of that environment as special or different (Runesson and Mok, 2005). A fundamental belief in the Learner's Perspective Study (LPS) was that "international comparative studies are likely to reveal patterns of practice less evident in studies limited to a single country or community" (Shimizu, et al., 2009, p.11). A significant stimulus for comparative classroom studies was the seminal work undertaken in the TIMSS Video Study. The initial stage of TIMSS Video Study involved only 3 countries and was reported in the book "The Teaching Gap" (Stigler \& Hiebert, 1999) and later extended to 7 countries in the follow-up TIMSS 1999 Video Study (Hilbert, et al., 2003). When the originators of the LPS project, Clarke, Keitel and Shimizu started the project, they were motivated to a significant extent by a perceived need to complement the survey-style approach characteristic of the work of Stigler and his co-workers with a more in-depth approach that captured more of the perspective of the learner. The positioning of the research methodology for the Learner's Perspective Study (LPS) was that the design must be "sufficiently sophisticated to accommodate and represent the multiple perspectives of the many participants in complex social settings such as classrooms. Only by seeing classroom situations from the perspectives of all participants can we come to an understanding of the motivations and meanings that underlie their participation. Our capacity to improve classroom learning depends on such understanding." (Clarke, 2006, p.15) The design of the project centred on three key requirements: (i) the recording of interpersonal conversations between focus students during 
the lesson; (ii) the documentation of sequences of lessons, ideally of an entire mathematics topic; and, (iii) the use of post-lesson interviews with the teacher and selected students to identify the intentions and interpretations underlying the participants' statements and actions during the lesson.

Essential features of the LPS research design are (i) the on-site mixing of the images from two video cameras to provide a split-screen record of both teacher and student actions and (ii) the use of the technique of video-stimulated recall in interviews conducted immediately after the lesson to obtain participants' reconstructions of the lesson and the meanings which particular events held for them personally. A third camera recorded "corporate" student practices - that is, the practices common to the whole class group. Two students were interviewed after each lesson. Each teacher participated in three video-stimulated interviews and completed two substantial questionnaires before and after videotaping, as well as a shorter questionnaire after each videotaped lesson. Copies were also made of student written materials, textbook pages, and worksheets used in class. With regard to the teachers, classes, and classrooms being sampled: eighth grade lessons were recorded in three classrooms for each country, for a minimum of ten consecutive lessons for each class/teacher. This produced a data set of at least 30 lessons for each country. The particular teachers whose classrooms were studied had been identified by the local members of the research team, in consultation with colleagues and members of the local mathematics education community, as engaging in "quality teaching practice" consistent with local curricular emphases.

The research teams in the LPS community are now based in universities in Australia, China, the Czech Republic, Germany, Israel, Japan, Korea, New Zealand, Norway, The Philippines, Portugal, Singapore, South Africa, Sweden, the United Kingdom and the USA (http://www.lps.iccr.edu.au/). The LPS database documents the teaching of sequences of lessons and provides a rich integrated data set generated from very diverse education systems, values and cultures. The dataset allows researchers to reconstruct accounts of lesson events by combining the video-records, with the teachers' and the students' perspectives as expressed in post-lesson interviews. There is a continual generation of new findings and insight accompanied by the addition of new international partners leading to the steady expansion of the dataset and even greater potential for cross-cultural analysis. While the international partnerships afforded by the LPS provides opportunities for contextualized understanding of the systems, issues and tensions from the perspectives of both insiders and outsiders, the international collaboration has also contributed to the creation of a new agenda for parallel and comparative analysis of classroom practice in mathematics classrooms internationally.

\section{STORIES FROM THE INSIDERS: CASES WITH DIFFERENT FOCUS}

In the first book in the LPS series, Mathematics Classrooms in Twelve Countries: The Insider's Perspective (Clarke, et al., 2006), researchers from 12 participants were invited to write up chapters based on the data within their own countries. The meaning of "the insider's perspective" was conceived as two-fold in the preparation of the book. Firstly, the writers of each chapter are considered as insiders as their analysis and writing are based on the data generated from their own cultures and school systems; and they voiced their analyses from 
that position. Secondly, the other "insiders" were the participants in the classrooms, i.e., the teachers and the students, and the LPS analyses have taken into account of these "insider" voices available in the dataset.

How teaching and learning take place inside the classroom is a complex system comprising different interacting components that include the teacher, the students, the curriculum, the learning materials and the interactions in the activities in the lesson. It is difficult to describe all details in full, but, in fact, the details in each aspect matter significantly. When each researcher constructed their insider's chapter, they looked into the LPS data collected from their home country and reported the analysis of an important aspect according to their own perspectives. The resulting reports in the book, taken as a whole, therefore do not represent a consensus on how classrooms should be viewed. More importantly, the differences represent what researchers from each community saw as relevant and significant. The complementary conglomerate vision created by the efforts of the international team gives a rich and culturally nuanced picture of classroom practice. Here, in this section, I summarize a few examples.

Keitel (2006) analysed "setting a task" in German schools. Keitel and her team analysed classroom practice in the three LPS German schools to find out what kind of tasks were set, what were the differences in how the teachers set tasks, and how these differences (if there were any) might affect the students' learning of mathematics as a school subject and an important scientific and social enterprise. Their basic assumptions were that tasks had a double function: they represented the means for teaching and assessing, and also provided a mechanism for securing objective standards of evaluation. In the words of Keitel (2006), "a strange amalgam of setting up various tasks for conflicting purposes developed. The teaching and assessing on one hand means that tasks are designed to develop performance abilities, to prepare for assessment, and to serve as means of assessment that evaluates these abilities" ( $p$. 42). Keitel compared the lessons of two teachers G1 and G2. The teacher G1 regularly started with a series of tasks for the purpose of practicing and memorizing specific algebraic rules and he told the students repeatedly that it helped them prepare for the regular assessment tests. Therefore, the students followed him and were accustomed to this kind of routine task. The students mostly worked individually with little collaboration. There was a lesson in which the teacher introduced a non-routine question aiming to link algebra with geometry through a problem involving a proof of the algebraic expansion of the square of $(a+b)$ via the area of the display of squares and rectangles. However, analysis showed "the overwhelming emphasis on mastering routines and algorithms seemed to be extended to all parts of mathematics classroom practice in G1 and overshadowed even those events that actually broke with routines and tried to offer a new kind of insight and enlightenment." In contrast, the teacher G2 structured the lessons less formally and regularly. Although he also used formal routine tasks, his lesson plans allowed more improvisation and he was more supportive of students' self-initiated activities and collaboration. Students in this class had more time for discussion in groups and for the presentation of their ideas and methods. In the students' interviews, they reported that they liked working together and they felt that the collaborative activities were more demanding, as they had to find new ways on their own and they appreciated that as "thinking mathematically" (p. 52). 
Hino (2006) reported on the role of seatwork in three Japanese classrooms, based on the analysis of the LPS data from three Japanese classrooms (J1, J2 and J3). "Seatwork" is an organizational segment in the lesson in the analysis of work in the Third International segment and is examined in terms of its frequency, length and pattern and process (Stigler et al., 1999). By the definition of Stigler and co-workers (1999), seatwork activity refers to the period in the lesson when students work on assigned tasks independently or in small groups. Hino (2006) examined the teacher's support for individual students during seatwork, the role of seatwork in the development of the lesson and its relationship to students' learning. In Japan, seatwork is called "Kikan-Shido" meaning instruction at students' desks that includes the teacher's purposeful scanning of the individual student's problem solving process (Shimizu, 1999). The analysis by Hino (2006) showed that seatwork was important in Japanese lessons. Following the seatwork activity, the teacher used the students' work for a variety of purposes including: eliciting and making use of their work, eliciting their mistakes, eliciting their puzzlement, eliciting opposing solutions, pointing out different solutions and giving explanation, pointing out difficulties and giving explanations, taking up students' questions and making their way of thinking visible to the group. For the Japanese lessons in the three classrooms, seatwork occurred before presenting the main content. This sequencing let the teacher support the individual students by orchestrating the development of the lesson after the seatwork activity, drawing on the teacher's knowledge of the students' understanding, obtained from observation during the seatwork activity. From the analysis of the students' interviews, seatwork activity was viewed as one of the most important for the activity for two reasons. Firstly, it provided time to exchange information and opinions with their neighbours and to think about the problem together. Secondly, it helped the students' thinking and understanding in the later part of the lesson.

Wood, Shin and Doan (2006) attempted to find out how the objectives of mathematics education reforms were realized in three US classrooms. The analysis of the Third International Mathematics and Science Study (TIMSS) 1995 and 1999 Video Study data (e.g., Jacobs et al., 2006 and Leung, 2005) reported that the US lessons remained "traditional." Despite the findings of TIMSS and TIMSS Video Study, Wood and co-authors (2006) argued that the analysis of the LPS data of the US classroom for the evidence of curriculum elements was essential for two reasons: (1) the three US classes (US1, US2, US3) in the LPS data contained features that were important in the realisation of the current curriculum standards in mathematics in the US, and (2) the LPS data consisted of a sequence of consecutive lessons that could provide greater detail about the nature of teaching in the US classrooms than could be obtained from the TIMSS Video Study. Moreover, an essential feature was that the data provided insights into both the teachers' practices and the learners' practices. They analysed the data collected from the three US schools, focusing specifically on US3, to examine how these classrooms realised the goals of the curriculum in school mathematics instruction. They applied, in the analysis, a conceptual framework with two dimensions: student participation and student thinking, aiming to describe the differences among the three classrooms in terms of the relationship between these two dimensions. Close examination of the teaching and learning in the class US3 showed many of the characteristics of reform. Students did work in groups and were given autonomy to check their homework and to clarify what they did not 
understand. However, the quality of mathematical experiences was generally focused on procedural solutions for the problems. The teacher spent the majority of the lesson in routine activities such as taking attendance and checking homework. Consequently, listening to students was not an essential activity, because a majority of the teacher's actions did not require knowledge of students' thinking. Illustrating with a class episode, Wood and co-authors described, "In this class, the teacher rarely provided mathematical reasons for the steps in the procedures she presented. Instead, when students encountered difficulties executing the procedures, she relied on the authority of the text (often explicitly as "they said") for definitions and created rules, such as "you can't do that", to justify the steps in a procedure" (p. 82). Based on their analysis, Wood and her co-workers further argued that "the gap between research on mathematics teaching and learning practices and current school instruction still exists after nearly two decades; it is clear from the analysis of this data that one reason is that the degree of change is more far-reaching than initially perceived" (p.83).

Mok (2006) addressed the issue of teacher dominance by exploring the teacher's and the students' perspectives on their mathematics lessons as revealed in the Chinese data. Mok aligned the teaching in the lessons of a Shanghai classroom (SH2) with the teacher's self-evaluation of the lesson and the students' perspectives of their lessons. The findings showed that the teaching revealed features consistent with the prevailing image found in the studies of Asian classrooms (e.g., Mok and Morris, 2001; Park and Leung, 2006); that the teacher takes up a very influential role. The teacher showed a deep understanding of the subject and attempted to guide his students to understand the same level of detail. The teacher explicitly said that his teaching was not traditional and that a central thesis was to provide students the opportunity to comprehend and to think. From the analysis of what happened in the lessons, it was clear that there were opportunities for discussion, but the teacher exercised much control through the deliberate design of the classroom tasks. The teacher encouraged students to express their ideas in their own words but he at the same time emphasized the use of standardized mathematical language. From the analysis of the students' interviews, the students showed a deep appreciation and respect for their teacher and the learning of mathematical knowledge. Their central focus was on the content of the lesson and the teacher's actions. The results showed there was a correspondence between the teacher's expectations and the students' expectations for the lessons, while there was a mismatch between the teacher's rhetorical affirmation of western pedagogical models and his pragmatic practice based on his interpretation and synthesis of different teaching models.

A few other examples include:

- Kaur, Kiam and Hoon (2006) studied the role of the textbook and homework in two Singapore classrooms. Textbooks in Singapore were produced commercially and competed for official adoption by the Ministry of Education (MOE). Therefore, the content of textbooks matched very closely with the mathematics syllabus reflecting the expectations of the national standard.

- Ulep (2006) studied how a Philippines teacher used a motivational strategy called "Ganas" in the teaching and also investigated the influence of "Ganas" on the students" 
learning. The case study demonstrated how improvisation in a teacher's strategies made an impact on the classroom practice.

- Williams (2006) studied how the Australian lessons might have supported creative mathematical thinking.

- Park and Leung (2006) studied the characteristics of the Korean mathematics classroom within the framework of variation and interpreted the results in terms of the underlying cultural values that shared with other East Asian countries.

\section{MAKING CONNECTIONS BY COMPARING LESSON EVENTS}

When one is constantly in a familiar education system, one may easily take things for granted and lose sight of the underlying value system. When comparisons of education systems at different levels are made, one often sees some characteristics as less or more prominent than would be apparent without comparison, bringing about a better understanding of the affordance and limitation within a culture and between cultures. Therefore, one of the objectives for international comparison is to learn from other systems about teaching and learning so that one can reflect on one's own system (Runesson and Mok, 2005). Comparison of cases between different cultures and backgrounds is the principal approach in the analyses reported in the second published LPS book. The fundamental questions addressed are what to compare and how to compare.

In the second book in the LPS series, Making Connections: Comparing Mathematics Classrooms Around the World (Clarke, Emanuelsson, Jablonka \& Mok, 2006), it is argued in the complementary approach employed in the LPS research design that lesson structure can be studied at three levels at least: whole lesson, topic and lesson events. In the first half of the book, "Lesson Events" (regularity in the form and function of types of the key lesson activities/events from which lessons are constituted) are used as an entry point for data analysis in relation to a variety of foci provided by different countries. The lesson events included: Beginning the lesson; Kikan-Shido (between desks instruction); Students at the front; Matome (summary of the lesson); and 'Learning task' lesson events.

Beginning of the lesson: Mesiti and Clarke (2006) examined the classroom practice of the beginning of the lesson over sequences of ten lessons from the LPS classrooms in the USA, Australia, Japan and Sweden. Purposeful selection was made to include data from culturally-distant settings. This particular lesson event was defined as beginning from the moment the teacher undertook the first communicative act for the whole class followed by the next ten minutes. The patterns of practice identified by the analyses represented coherent sets of actions regularly and/or effectively used specifically for the beginning of a lesson, possibly, but not necessarily, including the whole of the first ten minutes. Mesiti and Clarke identified the dominant components for beginning the lesson including: the pre-education component (administrative, organizational, pastoral care); the review component (focusing or warm-up, recap or run-through); the instruction component; the student practice component; the student assessment component (diagnostic, assessment); and the correction component (whole class, independent). 
Kikan-Shido: (O'Keefe, Xu and Clarke, 2006) investigated 'Kikan-Shido' or "between desks instruction" in eighteen classrooms located in Berlin, Hong Kong, Melbourne, San Diego, Shanghai and Tokyo. Kikan-Shido represented a very significant part of the activities supporting learning in the lessons. Four mutually exclusive principal functions within Kikan-Shido were identified: monitoring student activity, guiding student activity, organizational action and social talk. The analysis applied very detailed coding of subcategories for each of the principal functions and showed a range of variations between the practices of different teachers in different classrooms. Their findings suggested that the practice of Kikan-Shido can be purposefully used to distribute the responsibility for knowledge generation in the classrooms of competent teachers within the institutional and cultural norms constraining that practice.

Students at the front: Jablonka (2006) studied the students' activities when they were called to the front of the classroom, i.e., the side of the room on which the teacher's desk, the board, an overhead projector (OHP), a flip chart, or a screen was located. The selection of the six classrooms for analysis was based on the principle of maximizing contrast, but at the same time keeping a reasonable basis for comparison. The classrooms included two from Berlin, two from Hong Kong and two from San Diego. The forms taken by this lesson event might include one or several students working at the front at the same time. The functions of such variations on the "student at the front" lesson event included: an extra chance to get the teacher's comments, solving a new task in public, publicizing work, explaining work, providing a division of labour between teacher and students, and displaying work. The students might be writing solutions on the board, presenting an account of completed work, showing products of group work, or assisting the teacher in a demonstration.

Matome: Shimizu (2006) discussed the form and functions of the particular lesson event 'Matome' ("summing-up" in Japanese) in the LPS classrooms in Australia (Melbourne), Germany (Berlin), China (Hong Kong, Shanghai), Japan (Tokyo), and the USA (San Diego). He firstly analysed the form and functions of the event within the local contexts of the Japanese classrooms, followed by the comparison with corresponding events from the other countries. Matome in Japanese classrooms had the main features of teacher public talk, effective use of chalkboard and reference to the textbook. Matome was seen by teachers (and the researcher) to be indispensble in traditional Japanese classrooms for sharing and pulling together the students's solutions in the light of the goals of the lesson of the day. Matome was seen as very important in both the teachers' and students' perceptions in the Japanese classrooms. The analysis showed that the Australian teachers did not give a specific summary at the end of each lesson and they tended to wait until the end of the topic before delivering a summary. In the German classrooms, the teacher did give some summary or provided some general comments on students' procedure, but it did not seem to be common for the German teachers to conclude the lesson by discussing or summarising retrospectively what students had learned during the whole lesson. The US teacher made summaries like the Japanese examples, but the summary often appeared at the end of each activity, instead of at the end of a lesson. In contrast, the Asian classrooms showed both commonalities and differences. Japan and Shanghai showed similar engagement of the teacher and students in Matome-like events, whereas Hong Kong appeared to be different. 
'Learning task' lesson events: Mok and Kaur (2006) developed a definition for learning task lesson events (LT event). A learning task lesson event was defined as comprising not only the description of the task itself but also the actual lesson episode in which the teacher and the students engaged themselves in the task (that is, both the stated or written task and the subsequent and enfolding social activity). The class organization might be in any format such as whole class discussion or group work. In their definition, Mok and Kaur differentiated between a learning task and a practice item. A learning task was intended to teach the students something new and the sequence of learning tasks showed a coherent development of the object of learning, whereas, a practice item was mostly repetition of a taught skill. They compared 18 learning tasks from Australia, Germany, Hong Kong, Japan, Shanghai, Singapore and United States. The data were chosen by the researchers in each country from their LPS home data according to the developed definitions and examples. Three common dimensions emerged in the analysis: differentiation of the mathematical process, building a realistic context and building connections.

\section{LEARNING TASKS: A MAJOR VEHICLE IN MATHEMATICS LESSONS}

Mathematics tasks are the major vehicles in mathematics lessons (Hiebert \& Wearne, 1993, Shimizu, et al. 2010). The content of teaching and student activities in mathematics classrooms are organized around mathematical tasks. Therefore, the tasks are influential determinants of the implemented curriculum and the content of the lessons. However, how the students are engaged in the tasks is important. Depending how the teacher set the task and engaged the students in the task, the resulting learning experience can be very different. For example, a simple task of an algebraic formula $\left(a^{2}-b^{2}=(a+b)(a-b)\right)$ might be carried out in the form of exploratory pair work, where the students shared their reasons and argument in private in a German classroom, while the same task might be a teacher's example for demonstration in which the teacher gave strong guidance with limited whole class interaction in a Singapore classroom. Both can be effective ways for helping students' learning, but the students may learn different things from these very different experiences. Due to the restricted number of lessons sampled, there was no intention to claim any of the methods as being typical of the region or school system. Nonetheless, it is possible that one event is more likely to happen in a classroom than the other as a result of the values embedded in the teacher's beliefs and the long history of education practice in that particular school system or locality (Mok \& Kaur, 2006).

The third LPS book Mathematical Tasks in Classrooms Around the World (Shimizu, Kaur, Huang \& Clarke, 2010) is devoted entirely to research into the role of mathematical tasks. It enhances the understanding of the nature of tasks and provides alternative analytical frameworks for tasks and their role in mathematics lessons. The book carries on the LPS theme of examining established practice via international comparative research. In this book, focusing on the nature, role and implementation of mathematical tasks, the authors offer the reader a variety of images of classrooms from the countries participating in the Learner's Perspective Study.

In this section, the analysis reported in the chapter by Mok (2010) shows some images of Australian lessons and of Shanghai lessons, aiming to show how the enactment of 
mathematical tasks in different countries can be revealed through the lens of a learning model. Mok (2010) compared the learning task lesson events with purposeful sampling to compare the lessons from two classrooms with very different demographic background. Two consecutive Australian lessons (A1-L04 and A1-L05) on the topic of circumference of a circle and two Shanghai lessons (SH2-L02 and SH2-L03) on the topic of linear equations in two unknowns were compared. At the first level of analysis, all the tasks used in the lessons were classified and task events were classified as learning task lesson events (LT events) or non-learning task lesson events (non-LT events). The LT events included demonstration of new skills, explanation of new concept, investigation and solution of new problems. The non-LT events included review, repetition and practice. The first level of analysis gave a brief overall picture of the lesson, an illustrative example comparing A1-L04 and SH2-L02 is shown in Table 1. Although the two topics were different, both teachers began their lessons with review and followed with one focus LT event representing the major development of the topic of the day. At the same time, the comparison also showed that the Shanghai students had to do more tasks than the Australian students in one lesson - the Shanghai students had to do 4 practice items that was classified as non-LT event. Despite the purpose of the non-LT event being practice, the content of the items required the students to scrutinize and apply what was taught in the LT-event and the task provided a good pretext for consolidation of this new knowledge. In terms of similarity and differences in the routines of the lessons, the author wrote:

"It is very obvious that the pace in the Shanghai lessons was much faster than that in the Australian lessons. A Shanghai lesson always covered more tasks (including LT and non-LT) than an Australian lesson. Despite this difference, both teachers had a similar major routine of giving instruction about the task, followed by students carrying out the task and ending with the whole class paying attention to teacher-led interactive feedback." (Mok, 2009, p.124)

Table 1: A brief overall comparison between Lessons A1-L04 and SH2-L02

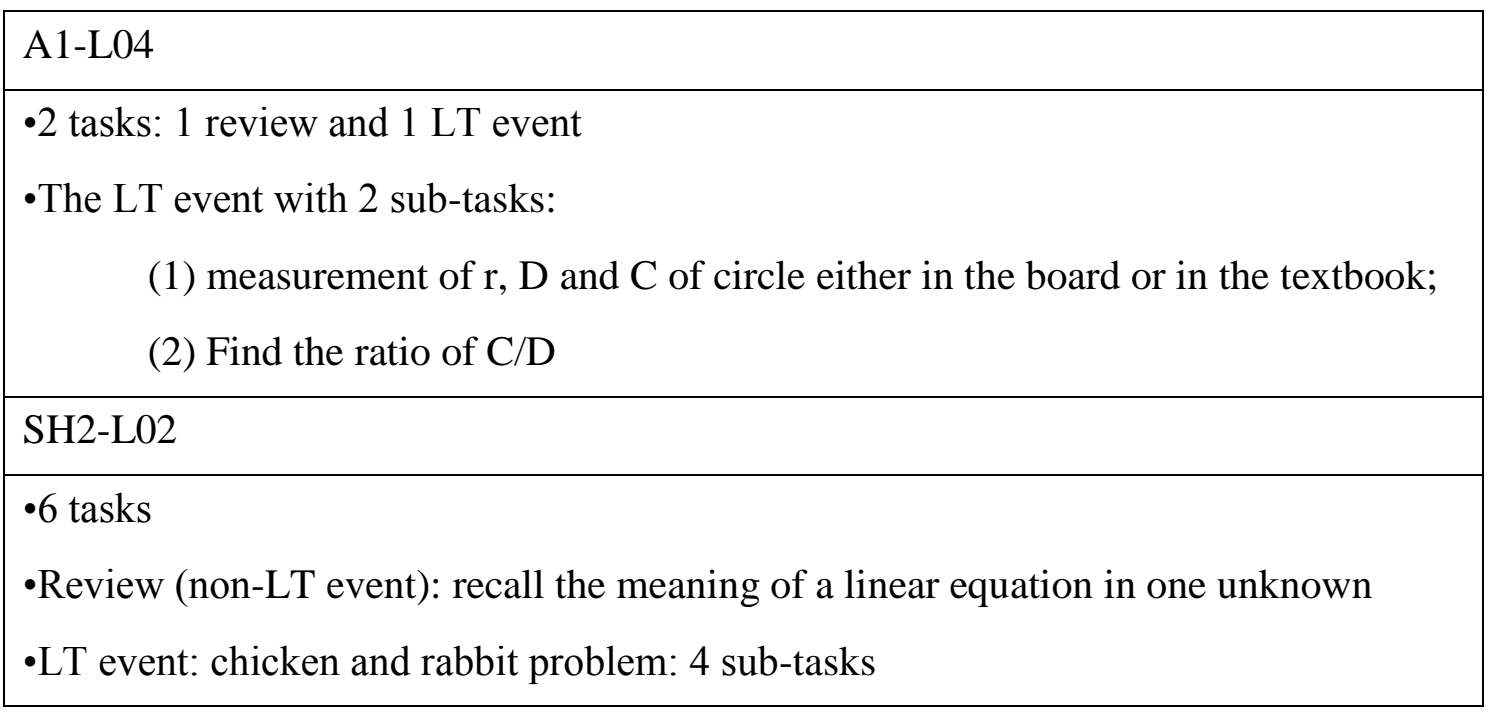


-4 students' practice (non-LT event):

-Non-LT1: rewrite an equation and write down the solution.

-Non-LT2: identify a linear equation into two unknowns from some give pairs

-Non- LT3 and non-LT4: determine whether a given pairs of numbers is a solution for a given equation or not.

The next stage of analysis is to look for a platform for investigating the nature of learning taking place. The first question that needs to be tackled is how to compare the teaching of different topics taken place in different cultural system and curriculum. Mok (2010) resolved this by making the cognitive process the focus for comparison. The chosen framework was a learning model based on the constructivist and social constructivist perspectives. In the framework, the elements of concrete preparation, cognitive conflict, metacognition and bridging were essential features for effective learning in the lessons.

"Concrete preparation covers a range of activities which include the provision of relevant technical vocabulary which will be useful for students' subsequent activities.

Cognitive conflict appears if children find a problem or task in which their methods and strategies appear not to work or to yield a contradiction, then the resulting mental conflict may challenge them to produce a higher-level strategy which does work.

Metacognition means reflecting on one's own thinking.

Bridging involves divergent thinking, where a person uses their imagination to invent other uses in contexts quite different from those in which they have learnt an idea or skill." (Mok, 2010, 121-122)

In the analysis of the selected lesson segments, the features of concrete preparation were explicit in the interaction elicited in the review activities. Often the teacher reviewed the concepts that would be needed for the following activities and the students answered readily. For example,

[A1-L05]

$\mathrm{T}$

Michael It's the distance around the outside of a circle.

$\mathrm{T}$

Sam

$\mathrm{T}$

S1

[to all] What is perimeter?

[to Michael] Michael.

[to Michael] Wonderful.

[to Sam] Sam.

The circumference.

Good girl. have we looked at?

The radius. [to all] What do we call the perimeter of a circle?

[to all] What other parts have we looked at, what other distances of a circle 
[SH2-L03]

T: $\quad$ Guess it: how many chickens and rabbits are there? There are $\mathrm{x}$ rabbits and $\mathrm{y}$ chickens in a cage. There are altogether twelve heads, and forty legs. How many rabbits and chickens are there in the cage?

Cobot: I'll set up two equations.

T: $\quad$ Oh, he said that he would set up two equations, tell me the first one.

Cobot: $\quad x$ plus y equals twelve.

$\mathrm{T}$ [Writing on the board] $\mathrm{x}$ plus y equals twelve.

Cobot: $\quad$ Four $\mathrm{x}$ plus two y equals forty.

Nonetheless, evidence of essential cognitive features did not suggest that these were necessarily exclusive of each other in interactions. For example, very often features such as cognitive conflict and metacognition were found in the class interaction when the teacher helped the students realize conflicting mathematical phenomenon and invited students to a deeper reflection.

For example, in lesson A1-L05, the teacher led the students to reflect upon the mathematics when inspecting two different ways representing a formula. This might happen spontaneously, when the teacher asked a why-question concerning the meaning of the variables. In this example, the teacher gave the students plenty of time to try their ideas and encouraged them to test their unconfirmed answers.

[A1-L05]

T: $\quad$ So I'm just going to substitute, instead of 'D', I'm just going to write 'Two R'. So that now says the circumference is equal to two pi two R. How does that match up with what I wrote just above? Are they the same?

Ben: [not audible]

T: $\quad$ Who said 'No'?

Ben: [not audible]

T: $\quad$ [to Ben] Ben, why not?

Ben: [not audible]

T: $\quad$ So they don't look identical, do they? Um, what does two pi $\mathrm{R}$ mean in expanded form?

Ben: $\quad$ Two times Pi times $\mathrm{R}$

T: $\quad \pi$ by two, then by R? Two by pi, then by R. So if I were to substitute a radius of five centimetres into this one, and into that one, what would I get? Would I get... You don't know? [to Stephen] Stephen?

Stephen: The first one is bigger than the top one. 
T: $\quad$ This one would be bigger than the top one? Why don't we try it, then? Michael, I'm going to need your help with the calculator, O.K.

On the other hand, the example found in the lesson by the Shanghai teacher $\mathrm{SH} 2$ showed a different pace. The teacher used the chicken and rabbit as a familiar context to guide the students through the scaffolding the mathematical ideas between a realistic context and abstract context. One main difference between this Shanghai example and the earlier Australian example is that the teacher moved to different levels of abstraction of the mathematical objects by employing a very compact and dense instructional mode, via his strategic scaffolding questions. The teacher invited the students to give the equations, suggest the different answers to the same equations, look back on their pairs of answers and reflect upon the context of generalization of equations.

[SH2-L02]

T: $\quad$ Please think about, think about the first question. The first question, ... in the cage, there are chickens, and rabbits as a sum of twelve, we have the equation $\mathrm{x}$ plus $\mathrm{y}$ equals to twelve, think about how many chickens are there, how many rabbits are there?

$\mathrm{T}:$

Tell me, what does the solution of linear equation in two unknowns mean? There are only three students willing to put up their hands! Okay, this student, tell us?

Catty: Among the numbers of solutions of the linear equation in two unknowns, each of the solution is the solution of the equation.

T: $\quad$ Okay, for this question, it is said that there are eleven pairs of solutions, if $x$ and $y$ indicates not real situation, that doesn't stand for rabbits, or chickens, so how many solutions are there for such an equation?

Class: Infinite.

The essential point is that consideration of the contribution of a task to classroom practice and to the learning of students must look beyond its simple written or spoken form and take into account the social interaction by which the task is performed in each classroom setting. Only be attending to the social enactment of the task can we come to understand its function in the mathematics classroom, which is so much more important than its simple mathematical form. This distinction between form and function was a pervasive comparative tool throughout LPS analyses in a wide range of investigative contexts.

\section{CONCLUSION}

What is mathematics classroom practice about? Mathematics classroom practice refers to the teaching and learning of mathematics that take place inside the classroom. It concerns the mathematical content, the organization, both the perceptions and activities of the teacher and the students, and the nature and content of the interaction in which the participants engage themselves (Laborde, 2006). 
What are international perspectives? International studies often involve comparison of learning achievement and outcomes. In the results of international studies such as the Program for International Student Assessment (PISA) or the Trends in International Mathematics and Science Study (TIMSS), the students' performances in some Asian countries consistently outperform the other countries. Therefore, it is tempting to make a claim to suggest the less-successful non-Asian countries would do well to adapt for their use the instructional practices of Asian classrooms. Nonetheless, such a claim may be problematic for there may be a "pseudo-consensus" imposed across systems (Keitel and Kilpatrick, 1999) that predetermines the criteria for comparative evaluation, while interpreting these results of comparison and Clarke and co-authors (2006) also point out that such assumptions have not given adequate attention to issues of cultural heterogeneity and differences in the students' experiences. Classrooms around the world differ in their underlying cultures, systems and beliefs. It is not possible to identify one model or system that may fit the purposes of all places. Nor is it right to say a certain kind pedagogical approach is better than the other in all instances and all settings. However, the search for differences and similarity is well justified.

In this paper, I discuss international perspectives about research on mathematics classroom practice based on the experience of the Learner's Perspective Study LPS. What may we learn about research on mathematical classroom practice from LPS?

A key concept in LPS is complementarity.

"Complementarity is fundamental to the approach adopted in the Learner's Perspective Study. This applies to complementarity of participants' accounts, where both the students and the teacher are offered the opportunity to provide retrospective reconstructive accounts of classroom events, through video stimulated post-lesson interviews. It also applies to the complementarity of the accounts provided by members of the research team, where different researchers analyse a common body of data using different theoretical frameworks." (Clarke, Kietel and Shimzu, 2006, pp. 4-5)

LPS demonstrates other facets and perspectives for international comparative studies of classroom practice. The idea of complementarity embedded in the project design and methodology is an essential feature. Also embedded in the design and methodology is the accommodation of diversity. The project recorded a sequence of consecutive lessons by the same teacher to assure the possibility to capture the variation due to the development of the topic or the diversity of learning opportunities created by the same teacher. The use of purposeful selection of competent teachers ensured capturing the practices by teachers who were locally recognized as representing the local conception of accomplished practice. Inside the classroom, both the teacher and the learners are the main agents and LPS has assured the recording of the voices of both parties in the data collection. The design of the project has allowed researchers to construct complementary accounts of what happens in the lessons from a variety of foci and theoretical perspectives. Similar to many international comparative studies, whenever the results of LPS are discussed in conferences and seminars, it is always of interest for the audience as to whether the reports observed in the classrooms in the study showed consistency of form and purpose, that distinguished the classrooms situated within 
one school system from other classrooms in different school systems, such as to suggest a culturally-specific character for a region. However, it has never been the intention of the LPS project to make claims about national typification of practice. Complementary and collaborative international efforts are the essential elements for activating the international perspectives. To that end, I conclude with the following quotes from the three books in the LPS series:

"The Learner's Perspective Study is guided by a belief that we need to learn from each other. The resulting chapters offer deeply situated insights into the practices of mathematics classrooms ... an insider's perspective." (Clarke, Kietel, and Shimizu, 2010)

"The comparisons made possible by international research facilitate our identification and interrogation of these assumptions. Such interrogation opens up possibilities for innovation that might not otherwise be identified, expanding the repertoire of mathematics teachers internationally, and providing the basis for theory development." (Clarke, Emanuelsson, Jablonka, and Mok, 2006)

"By making comparison possible between the classroom use of mathematical tasks in different classrooms around the world, the analyses reported in this book reveal the profound differences in how each teacher utilizes mathematical tasks, in partnership with their students, to create a distinctive form of mathematical activity." (Shimizu, Kaur, Huang, and Clarke, 2010)

\section{REFERENCE:}

Clarke, D. (2006). The LPS Research Design. In D. Clarke, C. Keitel, and Y. Shimizu, (Eds.) Mathematics Classrooms in 12 Countries: The Insiders' Perspective (pp. 15-36). Rotterdam: Sense Publishers B.V.

Clarke, D., Emanuelsson, J., Jablonka, E., and Mok, I.A.C. (Eds.) (2006). Making Connections: Comparing Mathematics Classrooms Around the World. Rotterdam: Sense Publishers B.V.

Clarke, D., Keitel, C. and Shimizu, Y. (Eds.) (2006). Mathematics Classrooms in 12 Countries: The Insiders' Perspective. Rotterdam: Sense Publishers B.V.

Hiebert, J., Gallimore, R., Garnier, H., Givvin, K., Hollingsworth, H., Jacobs, J., Chui, A., Wearne, D., Smith, M., Kersting, N., Manaster, A., Tseng, E., Etterbeck, W., Manaster, C., Gonzales, P., \& Stigler, J. (2003). Teaching mathematics in seven countries: Results from the TIMSS 1999 video study. Washington, DC: U.S. Department of Education, National Center for Education Statistics.

Hiebert, J., \& Wearne, D. (1993). Instructional tasks, classroom discourse, and students'learning in second-grade arithmetic. American Educational Research Journal, 30, 393-425.

Hino, K. (2006). The role of seatwork in three Japanese classrooms. In D. Clarke, C. Keitel, and Y. Shimizu, (Eds.) Mathematics Classrooms in 12 Countries: The Insiders' Perspective (pp. 59-74). Rotterdam: Sense Publishers B.V.

Jablonka, E. (2006). Students at the front: Forms and functions in six classrooms from Germany, Hong Kong and the United States. In Clarke, D., Emanuelsson, J., Jablonka, E., 
and Mok, I.A.C. (eds.) Making Connections: Comparing Mathematics Classrooms Around the World (pp. 107-126). Rotterdam: Sense Publishers B.V.

Kaur, B., Hiam, L.H. \& Hoon, S.L. (2006). Mathematics teaching in two Singapore classrooms: The role of the textbook and homework. In D. Clarke, C. Keitel, and Y. Shimizu, (Eds.) Mathematics Classrooms in 12 Countries: The Insiders' Perspective (pp. 99-116). Rotterdam: Sense Publishers B.V.

Keitel, C. (2006). 'Setting a task' in German schools: Different frames fro different ambitions. In D. Clarke, C. Keitel, and Y. Shimizu, (Eds.) Mathematics Classrooms in 12 Countries: The Insiders' Perspective (pp. 36-58). Rotterdam: Sense Publishers B.V.

Keitel, C., \& Kilpatrick, J. (1999). The rationality and irrationality of international comparative studies. Chapter 16 in G. Kaiser, E. Luna, \& I. Huntley (Eds.). International comparisons in mathematics education (pp.241-256). London: Falmer Press.

Laborde, C. (2006). Teaching and learning - An introduction. In F.K.S. Leung, K-D, Graf \& F.J. Lopez-Real (Eds.), The 13th ICMI Study: Mathematics education in different cultural traditions: A comparative study of East Asia and the West. (pp. 285-290). NY: Springer.

Mesiti, C. \& Clarke, D. (2006). Beginning the lesson: The first ten minutes. In Clarke, D., Emanuelsson, J., Jablonka, E., and Mok, I.A.C. (eds.) Making Connections: Comparing Mathematics Classrooms Around the World (pp. 47-72). Rotterdam: Sense Publishers B.V.

Mok, I.A.C. (2006). Teacher-dominating lessons in Shanghai: The insiders' story. In D. Clarke, C. Keitel, and Y. Shimizu, (Eds.) Mathematics Classrooms in 12 Countries: The Insiders' Perspective (pp. 87-98). Rotterdam: Sense Publishers B.V.

Mok, I.A.C. (2010). Comparison of learning task lesson events between Australian and Shanghai lessons. In Y. Shimizu, B. Kaur, R. Huang \& D. J. Clarke (Eds.), Mathematical tasks in classrooms around the world (pp. 119-145). Rotterdam: Sense Publishers B.V.

Mok, I.A.C. \& Kaur, B. (2006). Learning Tasks. In Clarke, D., Emanuelsson, J., Jablonka, E., and Mok, I.A.C. (eds.) Making Connections: Comparing Mathematics Classrooms Around the World (pp. 147-200). Rotterdam: Sense Publishers B.V.

Mok, I.A.C. \& Morris, P., (2001). The Metamorphosis of the 'Virtuoso': Pedagogic patterns in Hong Kong primary mathematics classrooms. Teaching and Teacher Education: An International Journal of Research and Studies, 17(4), 455-468.

O'Keefe, C., Xu, L. H. \& Clarke, D. (2006). Kikan-Shido: Between desks instruction. In Clarke, D., Emanuelsson, J., Jablonka, E., and Mok, I.A.C. (eds.) Making Connections: Comparing Mathematics Classrooms Around the World (pp. 73-106). Rotterdam: Sense Publishers B.V.

Park, K. and Leung, F.K.S. (2006). Mathematics lessons in Korea: Teaching with systematic variation. In D. Clarke, C. Keitel, and Y. Shimizu, (Eds.) Mathematics Classrooms in 12 Countries: The Insiders' Perspective (pp. 246-262). Rotterdam: Sense Publishers B.V.

Runesson, U. \& Mok, I. A.C. (2005). The teaching of fractions: A comparative study of a Swedish and a Hong Kong classroom. Nordic Studies in Mathematics Education, 10(2), October 2005. pp.1-15. 
Mok

Shimizu, Y. (1999). Aspects of mathematics teacher education in Japan: Focusing on teachers' roles. Journal of Mathematics Teacher Education 2, 107-116.

Shimizu, Y. (2006). How do you conclude today's lesson? The form and functions of 'Matome' in mathematics lessons. In Clarke, D., Emanuelsson, J., Jablonka, E., and Mok, I.A.C. (eds.) Making Connections: Comparing Mathematics Classrooms Around the World (pp. 127-146). Rotterdam: Sense Publishers B.V.

Shimizu, Y., Kaur, B., Huang, R. \& Clarke, D. J. (Eds.). (2010). Mathematical tasks in classrooms around the world. Rotterdam: Sense Publishers B.V.

Stigler, J., \& Hiebert, J. (1999). The teaching gap. New York: Free Press.

Ulep, S.A. (2006). 'Ganas' - A motivational strategy: Its influence on learners. In D. Clarke, C. Keitel, and Y. Shimizu, (Eds.) Mathematics Classrooms in 12 Countries: The Insiders' Perspective (pp. 131-150). Rotterdam: Sense Publishers B.V.

Williams, G. (2006). Autonomous looking-in to support creative mathematical thinking: Capitalising on activity in Australian LPS classrooms. In D. Clarke, C. Keitel, and Y. Shimizu, (Eds.) Mathematics Classrooms in 12 Countries: The Insiders' Perspective (pp. 221-237). Rotterdam: Sense Publishers B.V.

Wood, T., Shin, S.Y. \& Doan, P. (2006). Mathematics education reform in three US classrooms. In D. Clarke, C. Keitel, and Y. Shimizu, (Eds.) Mathematics Classrooms in 12 Countries: The Insiders' Perspective (pp. 75-86). Rotterdam: Sense Publishers B.V. 